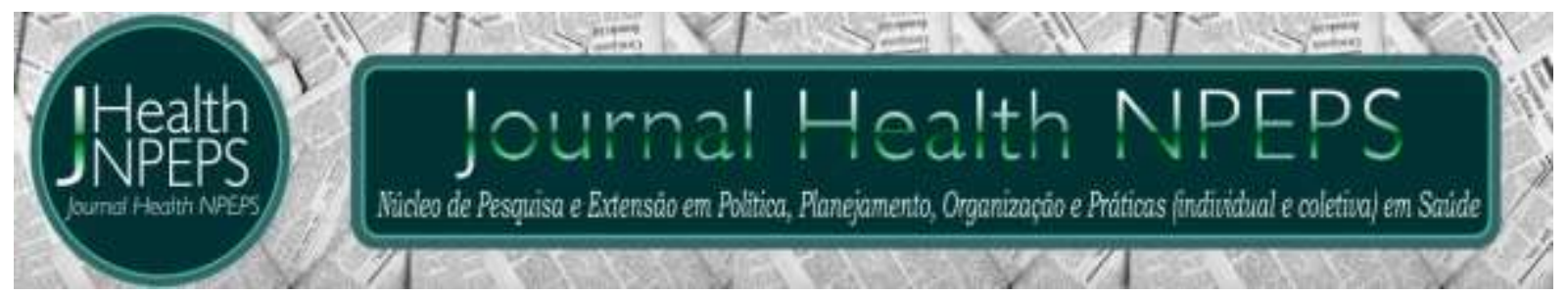

http://dx.doi.org/10.30681/252610102764

ARTIGO ORIGINAL

\title{
Intervención breve multicomponente de enfermería para reducir el consumo de tabaco: un estudio de factibilidad
}

\section{Brief multi-component nursing intervention to reduce tobacco consumption: a feasibility study}

\section{Intervenção breve de enfermagem multi-componente para reduzir o consumo de tabaco: um estudo de viabilidade}

\author{
Diana Aracely Almaraz Castruita ${ }^{1}$, María Magdalena Alonso Castillo ${ }^{2}$, Karla \\ Selene López García ${ }^{3}$, Marco Vinicio Gómez Meza ${ }^{4}$, Raquel Rodríguez Carvajal ${ }^{5}$
}

\begin{abstract}
RESUMEN
Objetivo: valorar la factibilidad de una Intervención Breve Multicomponete de Enfermería para reducir el consumo de tabaco. Método: estudio de factibilidad de un solo grupo y de mediciones repetidas, llevado a cabo de marzo a noviembre del 2017, en 3 áreas universitarias del área metropolitana de Monterrey, Nuevo León. Se utilizaron frecuencias y porcentajes y un análisis de comparación de medias ( $t$ de student) para dar respuesta a los objetivos planteados. Resultados: se mostraron cambios positivos en cuanto al número de cigarrillos fumados diariamente, la dependencia a la nicotina y los síntomas de depresión, ansiedad y estrés, los cuales disminuyeron significativamente al término de la intervención $(p<.005)$. Así mismo los participantes evaluaron el número, la frecuencia, la duración y el contenido de las sesiones como necesarios y estuvieron satisfechos con los resultados obtenidos. Consideraciones finales: dados estos hallazgos, y además de las diferencias significativas demostradas en las medidas de resultado, esta intervención se puede considerar factible para esta población y merece más estudio. Después de estos resultados, se comenzarán a realizar un estudio con mayor número de muestra.

Descriptores: Tabaquismo; Psicoterapia Breve; Atención Plena; Atención de Enfermería.
\end{abstract}

\section{ABSTRACT}

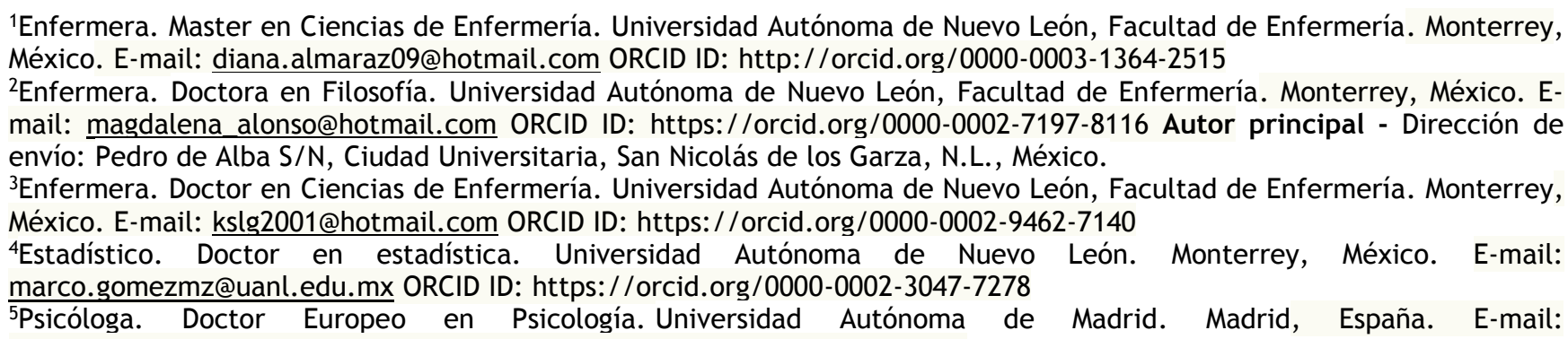
raquel.rodriguez@uam.es ORCID ID: https://orcid.org/0000-0001-9015-5425 
Objetive: assess the feasibility of a Brief Multicomponent Nursing Intervention to reduce tobacco consumption. Method: study of feasibility of a single group and repeated measurements, carried out from March to November 2017, in 3 university areas of the metropolitan area of Monterrey, Nuevo León. Frequencies and percentages and a comparison of means ( $t$-student) were used to answer the objectives. Results: there were positive changes in the number of cigarettes smoked daily, the nicotine dependence and the symptoms of depression, anxiety and stress, which decreased significantly at the end of the intervention ( $p$ <.005). The participants also evaluated the number, frequency, duration and content of the sessions as necessary and were satisfied with the results obtained. Final considerations: given these findings, and in addition to the significant differences demonstrated in the outcome measures, this intervention can be considered feasible for this population and deserves further study. After these results, a study with a larger sample number will begin.

Descriptors: Tobacco Use Disorder; Psychotherapy, Brief; Mindfulness; Nursing Care.

\section{RESUMO}

Objetivo: avaliar a viabilidade de uma breve Intervenção em Enfermagem Multicomponente para reduzir o consumo de tabaco. Método: estudo de viabilidade com um único grupo e medições repetidas, realizada de março a novembro de 2017, em três áreas universitárias da região metropolitana de Monterrey, Nuevo León. Utilizou-se frequências e porcentagens e uma comparação de meios ( $t$ de student) foram usados para responder aos objetivos. Resultados: houve mudanças positivas no número de cigarros fumados diariamente, a dependência de nicotina e os sintomas de depressão, ansiedade e estresse, que diminuíram significativamente no final da intervenção $(p<0,005)$. Os participantes também avaliaram $o$ número, a frequência, a duração e o conteúdo das sessões conforme necessário e ficaram satisfeitos com os resultados obtidos. Considerações finais: dados esses achados e, além das diferenças significativas demonstradas nas medidas de resultado, esta intervenção pode ser considerada viável para essa população e merece maior estudo. Após esses resultados, começará realizar um estudo com maior número de amostra.

Descritores: Tabagismo; Psicoterapia Breve; Atenção Plena; Cuidados de Enfermagem.

\section{INTRODUCCIÓN}

El consumo de tabaco es un factor de riesgo para seis de las ocho principales causas de mortalidad en el mundo así como para las cuatro Enfermedades No Transmisibles (ENT) más prevalecientes, responsables de casi dos tercios de las defunciones a nivel mundial. Se estima que casi siete millones de personas fallecen por esta causa anualmente, además se reportan 890.000 muertes debido a la exposición al humo de manera pasiva, de las cuales 170.000 son en niños ${ }^{1}$.

En la Región de las Américas el tabaquismo, es responsable del $80 \%$ de las defunciones y del 77\% de las muertes prematuras (muertes en personas de 30 a 70 años) de 
las que el $80 \%$ ocurrirán en países de ingresos bajos y medianos, con las implicaciones que esto tendrá para los sistemas de salud y las economías de esos países ${ }^{2-3}$. Si las tendencias actuales continúan, a partir de 2030 se estima que el tabaco será responsable de más de 8 millones de defunciones en todo el mundo 4 .

El consumo de tabaco no reconoce fronteras socioeconómicas y se ha demostrado el elevado costo que representa para todos los estratos de la sociedad. El costo directo de los sistemas de salud en América Latina por causas atribuibles al tabaquismo es de aproximadamente $\$ 33$ mil millones de dólares, equivalente a $0.5 \%$ del producto interno bruto (PIB) de la Región y a 7\% de todo lo que Latinoamérica gasta en salud al año. México no se queda atrás y el gasto atribuible al tabaquismo al sistema de salud le cuesta \$5 mil 110 millones de dólares, lo cual afecta no solo a la economía del país, sino también a la población con menos recursos económicos que vive en situación de vulnerabilidad 5 .

La nicotina es la principal substancia que conforma el tabaco, diferentes estudios han señalado que esta es la responsable del uso, mantenimiento y dependencia al consumo de tabaco y es un factor crucial para el fracaso durante los tratamientos ${ }^{6}$. La Asociación Americana de Psicología ha definido la dependencia a la nicotina como un síndrome que se caracteriza por una combinación de síntomas y comportamientos, que incluyen la tolerancia, los síntomas reiterados de abstinencia, el deseo (craving) y el uso compulsivo de la nicotina (uso del tabaco), entre otros?.

Así mismo, uno de los efectos característicos de la dependencia a la nicotina, es su uso reiterado para manejar el estrés y los trastornos emocionales. Investigaciones han encontrado diferentes determinantes de la motivación para el consumo de tabaco, entre los que destacan la búsqueda de la relajación ante el estrés de la vida, la costumbre o hábito, la presión de pares o del medio social, una baja autoeficacia para afrontar estos factores, el deseo de estimulación al estar deprimido o el reducir la ansiedad y el estrés ${ }^{8-}$ 10 .

Ante este último hallazgo la literatura hace evidente que la baja autoeficacia para resistir la influencia de pares/medio social, depresión, el estrés y la ansiedad son factores asociados al consumo de tabaco. Una hipótesis que explica dicha relación es que el uso del tabaco podría ser una forma de afrontamiento a estos factores, no obstante al dejar de fumar ya sea con o sin apoyo y tratamiento aunado a una baja autoeficacia para resistir la 
influencia de los pares/medio social, estos estados afectivos negativos (depresión, estrés, ansiedad) pueden influir en una recaída debido a que las personas desarrollan una fuerte dependencia al tabaco y pueden requerir de varios intentos y de multi tratamientos a largo plazo para alcanzar la abstinencia ${ }^{11-14}$, de manera que se necesitan intervenciones eficaces que puedan ayudar en el manejo de estos estados y así poder disminuir las tasas de fracaso y abandono de los tratamientos.

Uno de los profesionales de la salud interesados en ayudar a los pacientes a dejar de fumar es enfermería quienes mediante el consejo breve, con un refuerzo y algún tipo de seguimiento efectivo, como las líneas de ayuda telefónica, materiales educativos y de autoayuda tienen un fuerte potencial de factibilidad y efectividad ${ }^{15,16}$.

Debido a la complejidad de la adicción al tabaco y sus efectos nocivos sobre la salud se han desarrollado distintos tipos de intervenciones para dejar de fumar, las más utilizadas son la terapia cognitivo conductual y los tratamientos farmacológicos. La terapia cognitivo-conductual está basada en el asesoramiento, consejos breves, apoyo intensivo, reforzamiento de autoeficacia para resistir la tentación del consumo, entre otros. Mientras el tratamiento farmacológico consiste en el empleo de fármacos que controlen los síntomas que produce la abstinencia a la nicotina como lo es la Terapia de Reemplazo de Nicotina (TRN), entre otros ${ }^{17}$.

Según el National Cancer Institute (NCl), las estrategias de intervención para la cesación del uso de tabaco reflejan tasas de éxito muy fluctuantes, algunos resultados de estudios individuales sugieren que los tratamientos farmacológicos pueden ayudar a reducir los síntomas asociados a la cesación, sin embargo, estos medicamentos presentan múltiples efectos adversos, por lo que estos síntomas pueden influir en el abandono del tratamiento aunado a que estos fármacos no siempre están disponibles gratuitamente ${ }^{18,19}$.

Por otra parte las terapias cognitivo conductuales generalmente se centran en la enseñanza de habilidades de resistencia para evitar el consumo, mediante el aumento de la autoeficacia, la restructuración de estados afectivos asociados al consumo de tabaco, en la reducción del estrés (por ejemplo, relajación práctica), o en desviar la atención de las tentaciones, aprender estrategias cognitivas que reducen el estado de ánimo negativo y desarrollar mecanismos de apoyo social ${ }^{20,21}$, sin embargo, con frecuencia se aplican con un 
solo componente por lo que las tasas de éxito de esta modalidad de tratamiento son menores al 30\% a largo plazo 22 .

De esta manera, la evidencia de los síntomas de la abstinencia y el craving como perpetuadores de fumar, junto con la baja efectividad de los tratamientos actuales, pone de relieve la necesidad según el National Institute on Drug Abuse de proponer tratamientos multicomponentes innovadores para dejar de fumar como intervenciones que incluyan el consejo breve, reforzamiento de la autoeficacia de resistencia, materiales de orientación, intervenciones mente-cuerpo, seguimiento y monitoreo ya que estos parecen ser más exitosos ${ }^{23}$.

Las intervenciones mente-cuerpo se centran en las interacciones entre el cerebro, la mente, el cuerpo y el comportamiento que modifican el funcionamiento físico y promueven la salud ${ }^{24}$. Los componentes de estas intervenciones suelen ser la respiración profunda, la meditación y el yoga, los cuales han mostraron un aumento del $12.7 \%$ en su uso para mejorar algunos problemas de salud entre 2002 y 2007 en los Estados Unidos ${ }^{25,26 .}$

Dentro de las intervenciones mente-cuerpo destaca la Atención Plena (AP) 0 mindfulness, la cual tiene su origen en las tradiciones espirituales orientales que han sido modificadas y adaptadas para su uso clínico y terapéutico y se refiere a una forma de control de la atención desarrollada a través de prácticas de meditación ${ }^{27}$. La AP puede entenderse como atención y conciencia plena centrándose en el momento presente, de forma activa y reflexiva, destacando el focalizar la atención en las experiencias que se están viviendo en el momento presente, de forma intencionada, y aceptándolas sin juzgarse a sí mismos ${ }^{28}$.

Diversos estudios muestran que la AP ayuda al incremento en la atención, el bienestar subjetivo, la empatía y esperanza en pacientes con cáncer, entre otros ${ }^{29}$. Además ha mostrado su efectividad y se ha usado exitosamente en reducir el estrés, ansiedad y depresión en ámbitos tanto clínicos como no clínicos $^{30-32}$.

Algunos Ensayos Controlados Aleatorizados (ECA) sugieren que las prácticas de AP actúan sobre el eje Hipotalámico Hipofisario Adrenal (HHA), el cual se encarga de la regulación de los niveles de cortisol en sangre (hormona del estrés) ${ }^{33-35}$, así como a reducir el tono del sistema nervioso simpático, aumentar la actividad vagal ${ }^{36,37}$ y elevar los niveles de ácido y-aminobutírico cerebral (GABA) ${ }^{38}$. 
Así mismo la $A P$ se ha utilizado como una forma de tratamiento en distintos problemas de adicción a sustancias psicoactivas como el alcohol, la mariguana y el tabaco, mostrando resultados efectivos en la reducción y abandono del consumo y en el bienestar. Sin embargo, los autores recomiendan seguir utilizando esta intervención en diferentes modalidades y utilizar adicionalmente componentes motivacionales y de apoyo para aumentar las tasas de éxito 31 , 39-42.

Con referencia a lo anterior la investigación sobre la neurobiología de los trastornos por abuso de sustancias psicoactivas, entre ellas la adicción a la nicotina, indica que el uso crónico de drogas está asociado con un déficit en el control cognitivo dependiente del lóbulo prefrontal, que a su vez puede dañar el afecto y las vías inhibitorias $^{43}$.

La investigación en neuroimagen sugiere que la práctica de AP tiene la capacidad de modificar y mejorar los mecanismos de control cognitivo de conductas automáticas también conocidas como actividad neuronal descendente) por lo tanto podrían ayudar a reducir la reactividad hacia la nicotina y, consecuentemente, ayudar a dejar de fumar ${ }^{44-47}$.

De las formas de intervención de enfermería en adicciones que existen destaca el Consejo Breve para dejar de fumar (CB) el cual hace referencia a un intercambio de información y mensajes verbales, una actividad comunicativa entre profesional de la salud y paciente que promueve el desarrollo de capacidades para dejar de fumar ${ }^{48}$. Esta modalidad de intervención está diseñada con base en problemas reales y potenciales relacionados al tabaco y a los factores que motivan a los individuos a cambiar su comportamiento.

Según el NIDA 23 el Consejo Breve es una de las modalidades de intervención que ha mostrado ser efectivo cuando se usa con un enfoque de prevención y limitación de riesgos al: 1) mejorar los factores de protección y reducir los factores de riesgo; 2) tratar riesgos específicos a las características de la población (estrés percibido, depresión, ansiedad); 3) aumentar la competencia social con aptitudes de comunicación, relaciones con los compañeros, autoeficacia y técnicas para resistir la tentación del consumo; y 4) fortalecer el compromiso personal de mantener una conducta saludable es decir abandonar el consumo de tabaco. De esta manera resulta ser una herramienta que ayuda a potencializar 
los efectos y a aumentar las tasas de abstinencia y mantenimiento posteriores al abandono de la conducta cuando se usa en conjunto con otras formas de tratamiento ${ }^{49-51}$.

Conforme a lo anterior en el presente estudio, se evalúa de la factibilidad y la utilidad de una Intervención Breve Multicomponente de Enfermería que utiliza la Atención Plena fundamentada en los programas de Mindfulness Based Stress Reduction (REBAP) 52 y por el programa de Mindfulness Based Cognetive Therapy ${ }^{53}$ así como el programa de Consejería Breve IDEAS ${ }^{54}$.

A partir de ese contexto, el objetivo de este estudio fue valorar la factibilidad de una Intervención Breve Multicomponete de Enfermería para reducir el consumo de tabaco.

\section{MÉTODO}

El presente estudio de factibilidad de un solo grupo y de mediciones repetidas se realizó de marzo a noviembre del 2017, en 3 áreas universitarias del área metropolitana de Monterrey, Nuevo León. La población del estudio se conformó por adultos de ambos sexos de entre 18 y 59 años que fumaban y tuvieran intención de dejar el hábito, los criterios de inclusión fueron no tener algún diagnóstico médico de trastorno mental, no estar participando actualmente en algún programa para dejar de fumar y no consumir otra sustancia psicoactiva ilícita. Los participantes fueron reclutados a través de medios de comunicación, redes sociales y flayers colocados en puntos estratégicos de tres áreas universitarias. El muestreo se consideró no probabilístico por conveniencia y la muestra final estuvo conformada por 10 participantes.

El presente estudio se apegó a lo dispuesto en el Reglamento de la Ley General de Salud en materia de Investigación para la Salud ${ }^{55}$. Así mismo fue aprobado por los Comités de Ética e Investigación de la Facultad de Enfermería de la Universidad Autónoma de Nuevo León, con el número de protocolo FAEN-D-1258.

Una Cédula de datos sociodemográficos y de consumo de tabaco, la cual contenía datos generales del participante.

La Escala de Dependencia a la Nicotina Fagerstrom ${ }^{56}$ este instrumento tiene el fin de medir la dependencia a la nicotina relacionada con el consumo de cigarrillos por medio 
de seis interrogantes. Este instrumento ha sido validado en población mexicana con una confiabilidad de 0.56 a $.96^{57}$.

Escala de depresión, ansiedad y estrés (DASS-21) ${ }^{58}$ este instrumento tiene el propósito de medir la severidad de la alteración de los síntomas de depresión, ansiedad y estrés. Una alta puntuación en cada una de las dimensiones representa una mayor severidad de síntomas. Este instrumento ha sido utilizado en población mexicana ${ }^{59}$, con un alfa de Cronbach de .86 para toda la escala y un $46.6 \%$ de varianza explicada para el instrumento.

La Línea Base Retrospectiva (LIBARE) ${ }^{60}$ sirve para ayudar a consumidores de sustancias psicoactivas que inician un tratamiento a recordar su patrón de consumo. Mediante este método es posible obtener registros precisos del consumo, antes, durante y después del tratamiento. Diversos estudios psicométricos han mostrado que la Línea Base Retrospectiva de Consumo es un instrumento de medición adecuado con alfas que van desde .76 a .89 en población mexicana ${ }^{61}$.

La Escala de Evaluación Satisfactoria para el Participante. Esta escala fue desarrollada específicamente para este estudio por la autora principal a partir de la escala de Satisfacción percibida del Servicio Sanitario ${ }^{62}$ con el objetivo de evaluar el nivel de adecuación y satisfacción con respecto a la intervención. Esta consta de 7 reactivos que indagan sobre qué tan apropiados fueron el número de sesiones, la frecuencia, la duración y contenido, además indaga sobre cuales aspectos o sesiones de la intervención considera el participante como más importantes y menos importantes y si recomendaría o no la intervención a otras personas.

La Escala sobre Impresión Global de Cambio del Paciente ${ }^{63}$ tiene como objetivo medir la percepción del beneficio de los participantes de una intervención. Es una escala de siete puntos que califica el cambio en el estado general del participante desde el inicio de la intervención a través de 2 preguntas, la primera pregunta indaga sobre como el participante describe el cambio al finalizar la intervención y las respuestas van desde mucho mejor (7) a mucho peor (1), y la segunda pregunta averigua sobre el grado de cambio en general con la intervención en un compendio de 0 a 10 donde 10 es el máximo cambio posible, 5 es sin cambios y 0 es mucho peor que al inicio de la intervención. Las 
respuestas en el SGIC se han utilizado para determinar la importancia clínica de una intervención en diversas poblaciones y síntomas ${ }^{64,65}$.

Para el presente estudio se consideró que factibilidad sería el $80 \%$ de utilidad de la intervención según la opinión de los participantes (ítem 1 SGIC), más del 50\% se mostró satisfecho con la intervención (ítem 2 SGIC) y más del $80 \%$ de los ítems de la Escala de Evaluación Satisfactoria para el Participante se evaluaron como satisfactorios ${ }^{66}$. Los datos estadísticos se analizaron mediante el Statistical Package for the Social Sciences (SPSS), versión 17 para Windows. Se utilizó la estadística descriptiva, medidas de tendencia central y variabilidad y se realizó la prueba $t$ de student para calcular diferencias de cigarros fumados en un día típico al inicio y al final de la intervención.

En primera instancia se verifico la etapa de cambio o el interés de cambiar la conducta, posteriormente se les explicó a los participantes el objetivo de la intervención y se resolvieron sus dudas al respecto, luego se procedió con la obtención del consentimiento informado y el llenado de los cuestionarios para la medición basal. Cada participante recibió la intervención en su totalidad.

La intervención tuvo una duración de 10 semanas con sesiones semanales de una duración aproximada de 60-90 minutos por sesión en las cuales se utilizaron los programas Mindfulness Based Stress Reduction ${ }^{52}$ y el programa de Mindfulness Based Cognetive Therapy 53 así como el programa de Consejería Breve IDEAS 54 en conjunto. $A$ los participantes se les entrego un CD donde estaban grabadas las prácticas de AP para realizar en casa así como un manual de trabajo para llevar sus autoregistros así como orientaciones de reforzamiento que mantuvieran la motivación en el cambio de conducta. En la Tabla 1 se resume el contenido de cada una de las sesiones. La intervención se realizó en un consultorio silencioso con iluminación adecuada para llevar a cabo las prácticas de AP. La terapeuta siguió guiones detallados en un manual de facilitador que se realizó para la intervención para ofrecer la terapia individualizada de forma estandarizada para abordar aspectos específicos del consumo de tabaco.

Durante las semanas entre sesiones, se les pidió a los participantes que realizaran prácticas de AP y tareas enfocadas en el fortalecimiento de la autoeficacia para resistir el consumo de tabaco y que registraran sus experiencias que posteriormente fueron comentadas a la siguiente sesión. Una vez que se completaron las diez semanas de Journal Health NPEPS. 2018; 3(1):67-87. 
tratamiento, cada participante completó las escalas de evaluación post intervención. Además, al final del estudio se les proporciono los cuestionarios de SGIC y La Escala de Evaluación Satisfactoria para el Participante con la intervención, en esta segunda escala los participantes tenían un espacio libre para hacer sugerencias sobre áreas para mejorar relacionadas con el contenido o proceso de la intervención.

Los componentes de la intervención para cada sesión del estudio:

\section{Sesión 1}

Llenado de cuestionarios (medición inicial); Entrevista motivacional; Se abordó la forma general de trabajo; Entrega de CD de las prácticas de AP para realizar en casa así como un manual de trabajo para llevar autoregistros y orientaciones de reforzamiento.

\section{Sesión 2}

Revisión del LIBARE; Psicoeducación sobre las pautas de pensamiento que provocaban estados de ánimo negativos; Ejercicio de Atención Plena: escáner corporal, uva pasa.

\section{Sesión 3}

Revisión del LIBARE; Revisión de las prácticas y tareas en casa; Psicoeducación sobre consecuencias y beneficios en la salud del consumo/abandono del tabaco; Ejercicio de pensamientos y sentimientos; Ejercicio de Atención Plena: meditación sentado.

\section{Sesión 4}

Revisión del LIBARE; Revisión de las prácticas y tareas en casa; Establecimiento de meta de reducción; Psicoeducación: estrategias para el no consumo y evitar el consumo (estados de ánimo negativo); Ejercicio de Atención Plena: meditación de la escucha, ejercicio de espacio de respiración de 3 minutos (ER3M), estiramiento Atento.

\section{Sesión 5}

Revisión del LIBARE; Revisión de las prácticas y tareas en casa; Establecimiento de meta semanal de reducción; Indagación sobre la percepción actual del participante sobre su consumo y estrategias que tendría que utilizar para continuar con los avances; Ejercicio de Atención Plena: meditación de los sonidos y pensamientos.

\section{Sesión 6}

Revisión del LIBARE; Revisión de las prácticas y tareas en casa; Re-establecimiento de meta de reducción; Trabajo sobre la pauta interaccional del problema; Ejercicio de Atención Plena: meditación invitar a la dificultad, ER3M. 


\section{Sesión 7}

Revisión del LIBARE; Revisión de las prácticas y tareas en casa; Re-establecimiento de meta de reducción; Ejercicio de ventajas y desventajas del cambio; Ejercicio de Atención Plena: meditación invitar a la dificultad, ER3M.

\section{Sesión 8}

Revisión del LIBARE; Revisión de las prácticas y tareas en casa; Re-establecimiento de meta de reducción; Ejercicio de Atención Plena: ER3M.

\section{Sesión 9}

Revisión del LIBARE; Revisión de las prácticas y tareas en casa; Verificación del cumplimiento de meta; Prevención de recaídas; Ejercicio de Atención Plena: escáner corporal, vistazo retrospectivo del inicio y final de la terapia.

\section{RESULTADOS}

La media de edad de los participantes fue de 24.9 (DE=6.15), la edad de inicio de consumo de tabaco fue de 15.5 (DE=1.57), mientras que la media de años fumando diariamente fue de $6.8(D E=8.4)$. En cuanto al consumo de tabaco al pretest los participantes presentaron una media de 12 (DE=5.4) cigarrillos fumados por ocasión.

La Tabla 1 muestra los resultados en cuanto a variables sociodemográficas, en su mayoría los participantes fueron del sexo Masculino (70\%), con pareja (90\%), la mitad de ellos profesaba la religión católica (50\%), el 60\% había intentado dejar de fumar al menos una vez en la vida y el $80 \%$ pensaba que fumar lo ayudaba a relajarse.

Tabla 1. Variables Sociodemográficas.

\begin{tabular}{lcc}
\hline Variables & $f$ & $\%$ \\
\hline Sexo $\quad$ Masculino & 7 & 70 \\
$\quad$ Femenino & 3 & 30 \\
Estado Civil & & \\
$\quad$ Con pareja & 9 & 90 \\
Sin pareja & 1 & 10 \\
Religió $\quad$ & 5 & 50 \\
$\quad$ Católica & 5 & 50 \\
Ninguna & & 60 \\
Haz intentado dejar de fumar alguna vez en la vida & 6 & 40 \\
No & 4 & \\
\hline
\end{tabular}




\begin{tabular}{lll}
\hline Que consigues con fumar & & \\
Me relaja & 8 & 80 \\
Me ayuda a tener confianza & 1 & 10 \\
Es un apoyo & 1 & 10 \\
\hline Nota: $f=$ frecuencia, \%=Porcentaje, $n=10$ & &
\end{tabular}

Los 10 participantes completaron la intervención en su totalidad, así mismo los 10 participantes indicaron estar satisfechos con el efecto de la intervención sobre la reducción de su consumo de tabaco.

En cuanto a la Escala de Impresión Global de Cambio del Paciente, para el reactivo número uno el 50\% de los participantes refirió sentirse mejor y el 50\% restante menciono sentirse mucho mejor con referencia al síndrome de abstinencia, necesidad de fumar, síntomas emocionales (estrés y ansiedad) y calidad de vida en general, relacionado con la necesidad de fumar al finalizar la intervención. En cuanto al reactivo número dos de la EGCP los 10 participantes experimentaron un nivel de mejora entre 8 y 10 (máximo nivel de mejoría).

Con referente a la Escala de Evaluación Satisfactoria para el Participante, en el reactivo que hace mención del número de sesiones el $90 \%$ de los participantes mencionó que eran suficientes mientras que el $10 \%$ menciono que eran medianamente suficientes. En cuanto a los reactivos que abordan la frecuencia de las sesiones el 100\% mencionó que eran apropiadas, con respecto a la duración y contenido de las sesiones el $90 \%$ mencionó que eran apropiadas y el $10 \%$ dijo que eran medianamente apropiadas.

Para los 10 participantes el número de cigarrillos fumados diariamente y los índices de los instrumentos DASS-21 y Fagerstrom en el pretest decrecieron significativamente en el postest. Los resultados se muestran en la Tabla 2.

Tabla 2. Resultados sobre el abandono del consumo de tabaco.

\begin{tabular}{lcccc}
\hline Variables & $\bar{X}$ & $D E$ & $t$ & $P$ \\
\hline Cantidad de cigarros fumados diariamente & & & & \\
Pretest & 12.0 & 5.52 & 7.04 & .001 \\
Postest & 1.2 & 1.98 & & \\
& & & & \\
Indice Fagerstrom & 19.0 & 15.9 & 3.53 & .006 \\
Pretest & 3.0 & 9.4 & & \\
Postest & & & & \\
Indice Dass-21 dominio estrés & 51.9 & 20.9 & 8.19 & .001 \\
Pretest & 14.7 & 12.3 & & \\
Postest & & &
\end{tabular}




\begin{tabular}{lcccc} 
Indice Dass-21 dominio ansiedad & & & & \\
Pretest & 37.4 & 24.9 & 4.02 & .003 \\
Postest & 10.0 & 7.5 & & \\
Indice Dass-21 dominio depresión & & & & \\
Pretest & 33.3 & 25.3 & 4.08 & .003 \\
Postest & 8.5 & 7.0 & & \\
\hline Nota: $X=$ Media, DE=Desviación estándar, $t=$ Estadístico t de student, $p=$ Valor de $\mathrm{p}$
\end{tabular}

Gran parte de la retroalimentación de los participantes para la mejora de la intervención fue positiva. Los participantes en su mayoría mencionaron que la práctica de meditación ejercicio de espacio de respiración de 3 minutos fue la parte más útil ya que les ayudaba a tomar un tiempo de reflexión antes de actuar impulsivamente consumiendo cigarrillos, así mismo los participantes en su totalidad refirieron que todas las sesiones eran importantes para lograr el cambio.

Con respecto a la duración de las sesiones y la frecuencia solamente un participante menciono que sería bueno aumentar el número de sesiones a 2 veces por semana, ya que el tiempo entre sesiones (cada semana) le parecía muy largo. El $100 \%$ de los participantes señaló que recomendaría a otras personas tomar el tratamiento, sin embargo tres personas adicionalmente mencionaron que lo más importante era tener un fuerte compromiso consigo mismo, un verdadero deseo y convicción de dejar el cigarrillo para poder lograr la meta y el bienestar.

\section{DISCUSIÓN}

El presente estudio fue diseñado con el propósito de probar la factibilidad de una Intervención Breve Multicomponente de Enfermería para disminuir el consumo de tabaco. Los resultados mostraron cambios positivos en cuanto al número de cigarrillos fumados diariamente, la dependencia a la nicotina y los síntomas de depresión, ansiedad y estrés, los cuales disminuyeron significativamente al término de la intervención. Así mismo los participantes evaluaron el número, la frecuencia, la duración y el contenido de las sesiones como apropiados y estuvieron satisfechos con los resultados obtenidos, se confirmó que el contenido fue válido y los participantes afirmaron que la intervención era recomendable 
para otras personas que desearan reducir o eliminar el uso de tabaco, por lo que este estudio de factibilidad cumplió su propósito,

Los resultados positivos demuestran que, al igual que otras intervenciones que tienen dentro de sus componentes intervenciones mente-cuerpo (atención plena) y consejería breve para reducir el consumo de tabaco y síntomas como depresión, ansiedad y estrés $39-42,48,67-69$. Esta intervención tiene la capacidad para producir resultados prometedores para el abandono del consumo de tabaco. Estas dos terapéuticas en conjunto tienen el potencial para mejorar estas dimensiones de la salud. Sin embargo, debido a que este fue un estudio de factibilidad principalmente para determinar si el contenido, número, frecuencia y duración de la intervención fue suficiente para abordar las medidas de resultado, se necesitan ensayos clínicos más rigurosos antes de poder realizar determinaciones definitivas del papel de una intervención multicomponente de enfermería de atención plena y consejería breve sobre el consumo de tabaco.

Por otra parte la retroalimentación positiva proporcionada por los participantes también mostró el apoyo del contenido de la intervención y la relevancia para la población del estudio. Los participantes respondieron positivamente a la intervención indicando que estaban satisfechos del efecto del tratamiento sobre su consumo de tabaco y todos sintieron un grado importante de cambio positivo en cuanto al síndrome de abstinencia, necesidad de fumar, síntomas emocionales (estrés, ansiedad y síntomas depresivos) y calidad de vida en general.

Dados estos hallazgos, y además de las diferencias significativas demostradas en las medidas de resultado, esta intervención se puede considerar factible para esta población y merece más estudio. Posterior a estos resultados, se desarrollara un estudio con mayor número de muestra y con un diseño metodológico riguroso.

\section{CONSIDERACIONES FINALES}

La atención plena y la consejería breve en conjunto fueron encontradas como una intervención multicomponente factible que muestra resultados prometedores en cuanto a la reducción del consumo de tabaco. Sin embrago se necesita más investigación antes de implementar un plan para integrar esta intervención en la práctica de enfermería. 
La mayor limitación de este estudio de factibilidad fue un número reducido de muestra y la distribución de la muestra donde la mayoría de los participantes eran del sexo masculino y tenían pareja lo que puede ser una falta de representación significativa de la población de estudio.

\section{REFERENCIAS}

1. Organización Mundial de la Salud. Tabaco. [Internet] 2018. [acceso 31 de enero del 2018]. Disponible en http://www.who.int/mediacentre/factsheets/fs339/es.

2. Mathers CD, Loncar D. Projections of global mortality and burden of disease from 2002 to 2030. PLoS Med. 2006; 3(11):e442.

3. Öberg M, Jaakkola Maritta S, Woodward A, Peruga A, Prüss-Ustün A. Worldwide burden of disease from exposure to second-hand smoke: a retrospective analysis of data from 192 countries. Lancet. 2011; 377(9760):139-46.

4. Organización Panamericana de la Salud. Informe Regional sobre Control de Tabaco. [Internet]. 2016. [acceso 23 de mayo del 2017]. Disponible en http: / / www.paho.org/hq/index.php?option=com_content\&view=article\&tid=11965\%3A2016regional-report-tobacco-control\&catid $=1279 \% 3$ Atobacco-control-

publications\&ltemid $=1188$ \&lang=es.

5. Pichon RA, Bardach A, Augustovski F, Alcaraz A, Reynales-Shigematsu LM, Teixeira-Pinto $M$, et al. Impacto económico del tabaquismo en los sistemas de salud de América Latina: un estudio en siete países y su extrapolación a nivel regional. Rev Panam Salud Pública. 2016; 40(4):213-21.

6. National Institute on Drug Abuse. Cigarettes and other Tobacco Products. [Internet]. 2015 [acceso 13 de enero del 2017]. Disponible en: https://www.drugabuse.gov/publications/drugfacts/cigarettes-other-tobacco-products.

7. American Psychiatric Association. Practice guideline for the treatment of patients with nicotine dependence. Washington: American Psychiatric Association; 1996.

8. Bennasar Veny M, Pericas Beltrán J, González Torrente S, Segui González P, Aguiló Pons A, Tauler Riera P. Self-perceived factors associated with smoking cessation among primary health care nurses: a qualitative study. Rev Latino-Am Enferm. 2011; 19(6):1437-44. 
9. Duarte C, Varela M, Salazar I, Lema L, Tamayo J. Motivaciones y recursos para el consumo de sustancias psicoactivas en universitarios. Hacia promoc salud. 2012; 17(1): 92104.

10. Leal B, Ocampo O, Cicero S. Niveles de asertividad, perfil sociodemográfico, dependencia a la nicotina y motivos para fumar en una población de fumadores que acude a un tratamiento para dejar de fumar. Salud ment. 2010; 33(6):489-97.

11. Calvete E. Género y vulnerabilidad cognitiva a la depresión: el papel de los pensamientos automáticos. Ansiedad estrés. 2005; 11(2):203-14.

12. Carmody T, Vieten C, Astin J. Negative affect, emotional acceptance, and smoking cessation. J Psychoactive Drugs. 2007; 39(4):499-508.

13. Domínguez RM, Pérez YV. Ansiedad, depresión y vulnerabilidad al estrés ante el diagnóstico reciente de diabetes mellitus tipo 2. Gac méd espirit. 2014; 16(3):24-31.

14. Moreno CA, Medina MM. Tabaquismo y depresión. Salud ment. 2008; 31(5):409-15.

15. Organización Mundial de la Salud. El Convenio Marco de la OMS para el Control del Tabaco. [Internet]. 2003. [acceso 10 de enero del 2017]. Disponible en http://www.who.int/fctc/text_download/es.

16. Organización Panamericana de la Salud. Las funciones de los profesionales de salud en el control del tabaco. [Internet]. 2004. [acceso 26 de enero del 2017]. Disponible en http://www.infodrogas.gub.uy/html/publicaciones/docs/control-tobacco2.pdf17.

17. Cañas A, Alba L, Becerra N, Mosquera C, Murillo R, Páez N. Eficacia y seguridad del uso de medicamentos para la cesación de la adicción al tabaco: revisión de guías de práctica clínica. Rev salud pública. 2014; 16(5):772-85.

18. Dutch Institute for Health Care CBO (DE). Guideline treatment of tobacco dependence. [Internet]. 2003. [acceso 27 de diciembre del 2016]. Disponible en http://www.treatobacco.net/en/uploads/documents/Treatment\%20Guidelines/Netherland s\%20treatment\%20guidelines\%20in\%20English\%202006.pdf.

19. Ministerio de Sanidad Servicios Sociales e Igualdad(ES). Plan Nacional sobre Drogas. [Internet]. 2007. [acceso 13 de enero del 2017]. Disponible en http://www.pnsd.msssi.gob.es/ciudadanos/informacion/tabaco/home.htm. 
20. Fiore C, JaÈn C, Baker T, Bailey W, Benowitz N, Curry S, et al. Treating Tobacco Use and Dependence: Update. Clinical Practice Guideline. Rockville: US Department of Health and Human Services; 2008.

21. Fiore C, Jaén R, Baker B, Bailey C. Treating tobacco use and dependence: update. Clinical practice guideline. Rockville: U.S. Department of Health and Human Services; 2010. 22. Law M, Tang J. An analysis of the effectiveness of interventions intended to help people stop smoking. Arch Intern Med. 1995; 155:1933-41.

23. National Institute on Drug Abuse. Preventing drug use among children and adolescents, a research-based guide for parents, educators, and community leaders. [Internet] 2003. [acceso 23 de marzo del 2017]. Disponible en https: //www.drugabuse.gov/sites/default/files/preventingdruguse_2.pdf.

24. National Center for Complementary and Alternative Medicine. Yoga: In Depth. [Internet] 2003. [acceso 4 de abril del 2017]. Disponible en.

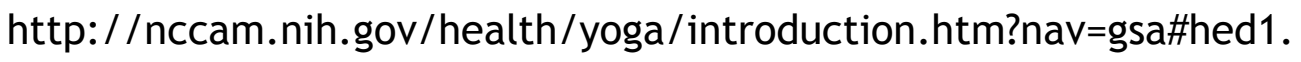

25. Barnes PM, Bloom B, Nahin RL. Complementary and alternative medicine use among adults and children: United States. Natl Health Stat. Rep. 2007; (12):1-23.

26. Nahin RL, Barnes PM, Stussman BJ, Bloom B. Costs of complementary and alternative medicine (CAM) and frequency of visits to CAM practitioners: United States. Natl Health Stat Rep. 2007; 30(18):1-14.

27. Baer RA. Mindfulness training as clinical intervention: A conceptual and empirical review. Clin Psychol Rev. 2003; 10(2):125-43.

28. Kabat-Zinn J. Full catastrophe living: Using the wisdom of your body and mind to face stress, pain and illness. Nueva York: Delacorte; 1990.

29. Shapiro S, Brown K, Thoresen C, Plante T. The moderation of Mindfulness-based stress reduction effects by trait mindfulness: results from a randomized controlled trial. Clin Psychol Rev. 2011; 67(2):67-77.

30. Arch J. Clinical trial of adapted mindfulness-based stress reduction versus group cognitive behavioral therapy for heterogeneous anxiety disorders. Behavior Res Theraphy. 2013; 51(4-5):185-96. 
31. Chiesa A, Serretti A. Mindfulness-based stress reduction for stress management in healthy people: a review and meta-analysis. J Altem Complement Med. 2011; 15(5):593600.

32. Fjorback LO, Arendt M, Ombol E, Fink $\mathrm{P}$, Walach $\mathrm{H}$. Mindfulness-based stress reduction and mindfulness-based cognitive therapy: a systematic review of randomized controlled trials. Acta Psychiatr Scand. 2011; 124(2):102-19.

33. Brand S, Holsboer-Trachsler E, Naranjo JR, Schmidt S. Influence of mindfulness practice on cortisol and sleep in long-term and short-term meditators. Neuropsychobiology. 2012; 65(3):109-18.

34. Vadiraja HS, Raghavendra RM, Nagarathna R, Nagendra HR, Rekha M, Vanitha N, et al. Effects of a yoga program on cortisol rhythm and mood states in early breast cancer patients undergoing adjuvant radiotherapy: a randomized controlled trial. Integr Cancer Ther. 2009; 8(1):37-46.

35. Vedamurthachar A, Janakiramaiah N, Hegde JM, Shetty TK, Subbakrishna DK, Sureshbabu SV, et al. Antidepressant efficacy and hormonal effects of Sudarshana Kriya Yoga (SKY) in alcohol dependent individuals. J Affect Disord. 2006; 94(1-3):249-53.

36. Bernardi L, Wdowczyk-Szulc J, Valenti C, Castoldi S, Passino C, Spadacini G, et al. Effects of controlled breathing, mental activity and mental stress with or without verbalization on heart rate variability. J Am Coll Cardiol. 2000; 35(6):1462-69.

37. Riley D. Hatha yoga and the treatment of illness. Altern Ther Health Med. 2004; 10(2):20-21.

38. Streeter CC, Whitfield TH, Owen L, Rein T, Karri SK, Yakhkind A, et al. Effects of yoga versus walking on mood, anxiety, and brain GABA levels: a randomized controlled MRS study. J Altern Complement Med. 2000; 16(11):1145-52.

39. Brewer J, Sinha R, Chen J, Michalsen R, Babuscio T, Rounsaville B, et al. Mindfulness Training and Stress Reactivity in Substance Abuse: Results from a Randomized, Controlled Stage I Pilot Study. J Subst Abuse. 2009; 30(4):306-17.

40. Souza I, Barros V, Gomide H, Miranda T, Menezes V, Noto A, et al. Mindfulness-based interventions for the treatment of smoking: a systematic literature review. Altern Ther Health Med. 2015; 21(3):129-40. 
41. Davis J, Manley A, Goldberg S, Stankevitz K, Smith S. Mindfulness training for smokers via web-based video instruction with phone support: a prospective observational study. BMC Complement Altern Med. 2015; 15:95.

42. Tang $\mathrm{Y}$, Tang R, Posner Ml. Brief meditation training induces smoking reduction. Proc Natl Acad Sci U S A. 2013; 110(34):13971-5.

43. Koob GF, Volkow ND. Neurocircuitry of addiction. Neuropsychopharmacology. 2010; 35: 217-38.

44. Allen M, Dietz M, Blair KS, van Beek M, Rees G, Vestergaard-Poulsen P, et al. Cognitiveaffective neural plasticity following activecontrolled mindfulness intervention. J Neurosci. 2012; 32(44):15601-10.

45. Cahn BR, Polich J. Meditation states and traits: EEG, ERP, and neuroimaging studies. Psychol Bull. 2006; 132(2):180-211.

46. Farb NAS, Segal ZV, Mayberg H, Bean J, McKeon D, Fatima Z, et al. Attending to the present: mindfulness meditation reveals distinct neural modes of self-reference. Soc Cogn Affect Neurosci. 2007; 2(4):313-32.

47. Lazar SW, Kerr CE, Wasserman RH, Gray JR, Greve DN, Treadway, MT, et al. Meditation experience is associated with increased cortical thickness. Neuroreport. 2005; 16(17):189397.

48. Alba LH, Murillo R, Castillo JS. Intervenciones de consejería para la cesación de la adicción al tabaco: revisión sistemática de la literatura. Salud Pública Méx. 2013; 55(2):196-206.

49. Finfgeld-Connett, D. Clarification of Social Support. J Nurs Scholarsh. 2005; 37(1): 4-9. 50. Hunt MK, Fagan P, Lederman R, Stoddard A, Frazier L, Griod K, et al. Feasibility of implementing intervention methods in adolescents worksite tobacco control study. Tob Control. 2003; 12(4):iv40-5.

51. Werch C. Preventive Alcohol Interventions Based on a Stage of Acquisition Model. Am J Health Behavior. 2001; 25(3):206-16.

52. Kabat-Zinn, J. La práctica de la atención plena. Barcelona: Kairós; 2007.

53. Segal Z, Williams M, Teasdale J. Terapia cognitiva basada en el mindfulness para la depresión. Barcelona: Kairos; 2007. 
54. Consejo Nacional Contra las Adicciones. Prevención de las adicciones y promoción de conductas saludables para una nueva vida. [Internet]. 2007. [acceso 13 de octubre del 2016]. Disponible en

http: //www.conadic.salud.gob.mx/pdfs/nueva_vida/nv1e_prevencion.

55. Secretaria de Salud (MX). Reglamento de la Ley General de Salud en Materia de Investigación para la Salud. [Internet]. 1987. [acceso 10 de enero del 2017]. Disponible en http: //www.salud.gob.mx/unidades/cdi/nom/compi/rlgsmis.html

56. Moreno CA, Villalobos GL. Psychometric Properties of the Fagerström Test for Nicotine Dependence in a Sample of Mexican Smokers. J Addict Nurs. 2017; 28(1):27-33.

57. Becoña IE. Bases teóricas que sustentan los programas de prevención de drogas. [Internet].1998. [acceso 20 de diciembre del 2016]. Disponible en http: //www.cedro.sld.cu/bibli/i/i4.pdf

58. Lovibond PF, Lovibond SH. The structure negative emotional states: comparison of the depression anxiety stress scale (DASS) with the beck depression and anxiety inventories. Behav Res Ther. 1995; 33(3):335-43.

59. Gurrola GL, Balcázar P, Bonilla MP, Virseda JA. Estructura factorial y consistencia interna de la escala de Depresión, Ansiedad y Éstres (DASS-21) en una muestra no clínica. Rev.Psico Cien Soc. 2006; 8(2):3-7.

60. Sobell LC, Sobell MB. Timeline Followback user's guide. Ontario: Addictions Research Foundation; 1996.

61. Torres L, Vásquez J, Medina-Mora M, Velásquez $\mathrm{H}$. Adaptación de un modelo de intervención cognoscitivo-conductual para usuarios dependientes de alcohol y otras drogas a población mexicana: un estudio piloto. Salud ment. 2005; 28(1):61-71.

62. Varela M J, Rial BA, García CE. Presentación de una Escala de Satisfacción con los Servicios Sanitarios de Atención Primaria. Psicothema. 2003; 15(4):656-61.

63. Guy W. Clinical Global Impressions. En: ECDEU Assessment Manual for Psychpharmacology, revised. Rochville: National Institute of Mental Health; 1976.

64. Hudson JI, Arnold LM, Bradley LA, Choy EH, Mease PJ, Wang F, et al. What makes patients with fibromyalgia feel better? Correlations between Patient Global Impression of Improvement and changes in clinical symptoms and function: A pooled analysis of 4 randomized placebo-controlled trials of duloxetine. J Rheumatol. 2009; 36(11):2517-22. 
65. Liu H, Tan AD, Qin R, Sargent DJ, Grothey A, Buckner JC, et al. Comparing and validating simple measures of patient-reported peripheral neuropathy for oncology clinical trials: NCCTG N0897 (alliance) a pooled analysis of 2440 patients. SOJ Anesthesiol Pain Manag. 2015; 2(2).

66. Cieslak A, Elkins G, Banerjee T, Marsack J, Hickman K, Johnson A, et al. Developing a Hypnotic Relaxation Intervention to Improve Body Image: A Feasibility Study. Oncol Nurs Forum. 2016; 43(6): E233-E241.

67. Elwafi HM, Witkiewitz K, Mallik S, Thornhill TA 4th, Brewer JA. Mindfulness training for smoking cessation: Moderation of the relationship between craving and cigarette use. Drug Alcohol Depend. 2013; 130(1-3):222-9.

68. García GL, Sansores R, Díaz V, Urdapilleta HE, González RG, PérezLE, et al. Impacto del consejo breve para dejar de fumar. Salud pública Méx. 2015; 57(5): 366-367.

69. Sanz PB, Miguel DJ, Anegon BM, García CM, Gómez E, Fernández DJ. Efectividad de un programa de consejo antitabaco intensivo realizado por profesionales de enfermería. Aten Prim. 2006; 37(5): 266-72.

Conflito de interesses: Os autores declaram não haver conflito de interesses.

Participação dos autores: Os autores declaram que participaram de todas as etapas do estudo (concepção, desenvolvimento do estudo, redação e revisão).

Como citar este artigo: Castruita DAA, Castillo MMA, García KSL, Meza MVG, Carvajal RR. Intervención breve multicomponente de enfermería para reducir el consumo de tabaco: un estudio de factibilidad. Journal Health NPEPS. 2018; 3(1):67-87.

Submissão: $22 / 02 / 2018$

Aceito: 15/06/2018

Publicado: 30/06/2018 